\title{
Temporal and subcellular distributions of Cy5.5-labeled hyaluronic acid nanoparticles in mouse organs during 28 days as a drug carrier
}

\author{
Chunmei Lin ${ }^{1,2, \uparrow}$, Saet Byeol Kim ${ }^{2, \dagger}$, Jung-Min Yon ${ }^{2, \dagger}$, Seul Gi Park², Lee Wha Gwon' ${ }^{2}$, Jong-Geol Lee ${ }^{2}$, \\ In-Jeoung Baek ${ }^{3}$, Beom Jun Lee ${ }^{2}$, Young Won Yun ${ }^{2, *}$, Sang-Yoon Nam ${ }^{2, *}$ \\ ${ }^{1}$ College of Chinese Medicinal Materials, Jilin Agricultural University, Changchun 130-118, China \\ ${ }^{2}$ College of Veterinary Medicine and Veterinary Medical Center, Chungbuk National University, Cheongju 28644, Korea \\ ${ }^{3}$ Asan Institute for Life Sciences, Asan Medical Center and University of Ulsan, Seoul 05505, Korea
}

(Received: August 24, 2017; Revised: December 14, 2017; Accepted: December 21, 2017)

\begin{abstract}
Temporal and subcellular distributions of hyaluronic acid (HA) as a degradable nanoparticle (NP) in animals were investigated to determine if HA-NP could be utilized as an appropriate drug delivery system. After mice were intravenously injected with $5 \mathrm{mg} / \mathrm{kg}$ of Cy5.5-labeled HA-NP sized 350-400 nm or larger HA-polymers, the fluorescence intensity was measured in all homogenized organs from $0.5 \mathrm{~h}$ to 28 days. HA-NP was greatly detected in spleen, liver and kidney until day 28, while it was maintained at low levels in other organs. HA-polymer was observed at low levels in all organs. HA-NP quantities in spleen and liver were reduced until day 3, but increased sharply between days 3 and 7, then decreased again, while their HA-polymers were maintained at low levels until day 28. In kidneys, both HA-NP and HA-polymer showed high levels after $0.5 \mathrm{~h}$ of administration, but steadily decreased until day 28 . According to ultrastructural analyses, HA-NP was engulfed in Kupffer cells of liver and macrophages of spleen and kidney at day 1 and was accumulated in the cytoplasm of kidney tubular cells at day 7. Overall, these findings suggest that HA-NP could be considered a desirable drug carrier in the liver, kidney, or spleen.
\end{abstract}

Keywords: drug delivery systems, hyaluronic acid, nanoparticles, pharmacokinetics, subcellular localization

\section{Introduction}

A drug delivery system (DDS) is defined as a formulation that improves the efficacy and safety of a drug by controlling the rate, time, and place at which drugs are released into the body. This process includes administration of the drug and the release across the biological membranes to the target site, where it is expected to exert therapeutic action. Among a number of new biotechnology-based therapeutics, nanoparticles (NPs) are considered to be important as drug delivery carriers [9]. Recent advances in nanotechnology in medicine have led to the development of biocompatible NPs for targeted therapy [17]. Properly developed NPs are expected to improve intracellular penetration, enhance absorption into target tissues, improve pharmacokinetic properties and to result in increased clinical efficacy and reduced toxicity.

Unfortunately, many systemically injected NPs are rapidly cleared from the blood stream by the reticuloendothelial system and the mononuclear phagocytic system, mainly through the liver, spleen, and bone marrow, resulting in a low thera- peutic index $[6,20]$. Accordingly, it is necessary to avoid NPs that clear rapidly $[1,6]$. NPs circulating for a long period of time should be also avoided as this accumulation may contribute to toxicity in target tissue [17, 23]. Therefore, development of NP that is maintained for a reasonable period is a necessary requirement for sufficient delivery to the desired target. Accordingly, biodegradable NPs must be selected to enable effective drug delivery devices in order to effectively deliver the drug to a target site and thus increase the therapeutic effect while minimizing toxicity [13, 23].

Hyaluronic acid (HA) has remarkable hydrodynamic characteristics, which has the viscosity and ability to retain water; therefore, it plays an important role in tissue homeostasis and biomechanical integrity [2]. HA also forms extracellular macromolecules that are important in the assembly of extracellular and pericellular matrices [25]. Owing to its biocompatibility and biodegradability, HA has been extensively investigated for biomedical applications such as tissue engineering [19] and drug delivery [16]. Because HA can specifically bind to various cancer cells that over-express CD44,

*Corresponding author

Tel: $+82-43-261-2596$, Fax: $+82-43-271-3246$

E-mails: ywyun@cbu.ac.kr (YW Yun), synam@cbu.ac.kr (SY Nam)

These authors contributed equally to this work. 
many studies have focused on the pharmaceutical applications of HA for anti-cancer therapeutics $[15,25]$. These properties are important factors in determining whether cells invade tissues during cancer progression [7, 12]. A previous study highlighted the key role of interactions between HA and tumor cells for new therapeutic strategies [25]. The relationships between HA receptors and tumor cells indicate that it may be possible to recruit HA for active targeting of carrier-loaded anticancer drugs [21]. However, even if HA is selected as a drug carrier based on these properties, the characteristics of biodistribution in the body are important parameters that must also be considered when designing and testing NPs as drug carriers. In addition, to achieve an effective level of NPs in the target tissue, targeted NPs should be transfused from circulating blood to the tissue of interest and bind to their molecular target as a first step in nanomaterial retention or cellular internalization $[17,20]$.

Most studies of HA-NP have described the distribution of only one size of particle [4]. However, the size of NPs can be a significant determinant of particle distribution, as shown for gold NPs [5], silica NPs [8, 27], and silver NPs [14]. The present study was conducted to confirm the accumulation and distribution of biodegradable HA-NP according to size and determine whether HA-NP could be utilized as an appropriate drug carrier in DDS. To accomplish this, the in vivo biodistribution and subcellular targeting patterns of HA-NPs and HA-polymers were observed based on the fluorescence intensity of Cy5.5-labeled NPs intravenously injected into mice and by transmission electron microscopy (TEM) images in cells.

\section{Materials and Methods}

\section{Animals}

Six-week-old male ICR mice were purchased from KOATECH (Korea) and allowed to acclimatize for 1 week before starting the experiment. Seven-week-old male mice weighing $20 \pm 2 \mathrm{~g}$ were used in the experiment. Animals were bred at a room temperature of $21 \pm 2^{\circ} \mathrm{C}$, a relative humidity of 55 $\pm 10 \%$, an air ventilation rate of 10 cycles per hour, and a 12:12 h light/dark cycle. The animals were fed standard mouse chow (Samyang, Korea) and tap water ad libitum throughout the experimental period. All animal experiments were performed in accordance with the Guide for Care and Use of Animals (Chungbuk National University Animal Care Committee, CBNUA-608-13-01).

\section{Preparation of nanoparticles}

HA-NP and HA-polymers used in this experiment were provided by Dr. Kwangmeyung Kim, Korea Institute of Science and Technology (Seoul, Korea). The HA-NPs were spherical in shape and their sizes were in the range of 350 to $400 \mathrm{~nm}$, depending on the degree of substitution of $5 \beta$ cholanic acid [4]. For animal tests, HA-NPs were labeled with Cy5.5 to visualize their biodistribution in vivo as previ- ously described [4]. Briefly, amphiphilic HA-5b-cholanic acid conjugates were chemically modified with acid dihydrazidemodified Cy5.5. The content of Cy5.5 molecules in the conjugate was fixed to 3.2 weight percentage as determined using a UV/VIS spectrophotometer at $680 \mathrm{~nm}$.

\section{Experimental design}

Mice were divided into two groups: HA-NP and HA-polymer. Mice were intravenously injected through the tail vein with a single dose of $5 \mathrm{mg} / \mathrm{kg}$ of Cy5.5-labeled HA-NP or HA-polymer. The injections were tolerated and no adverse effects were observed throughout the experiment. At $0.5 \mathrm{~h}$, $2 \mathrm{~h}, 4 \mathrm{~h}, 1$ day (d), $3 \mathrm{~d}, 7 \mathrm{~d}, 14 \mathrm{~d}$, and $28 \mathrm{~d}$ after injection, mice ( $n=6$ per time group) were anaesthetized with ether and subsequently euthanized by drawing blood from the abdominal aorta. Their final body weights were then measured and the livers, spleens, kidneys, lungs, hearts, brains, testes, epididymides, submandibular glands, small and large intestines, and thymuses were collected for fluorescence intensity measurement. Moreover, a portion of the organs were fixed and trimmed for histopathological examination and TEM analysis.

\section{Analysis of fluorescence intensity}

The biodistributions of HA-NP and HA-polymer or free Cy5.5 without HA in the examined organs were comparatively investigated by measuring the fluorescence intensity over different time periods for 28 days. For measurements, each discretized tissue was homogenized, after which the sample concentrations were measured in the tissues. To exclude the influence of the organ weight, all fluorescence intensity results were compared after calculation by the unit organ weight. Three hundred milligrams of collecting organs were put into $600 \mu \mathrm{L}$ of phosphate-buffered saline ( $\mathrm{pH} 7.4)$, after which the tissues were homogenized at $0.9-6 \times \mathrm{g}$ for 5 min using a homogenizer (PT 2100; Kinematica, Switzerland). The homogenized tissues were then placed on a black plate for fluorometer and fluorescence intensity was measured using the FLx800 Fluorescence Microplate Reader (BioTek, USA) at an excitation of $630 \mathrm{~nm}$ and emission of $730 \mathrm{~nm}$.

\section{Histopathological examination}

The livers, kidneys, and spleens were removed and fixed in $10 \%$ formalin. For histological examination, paraplast-embedded tissues were sectioned at $4 \mu \mathrm{m}$, then were stained by the routine hematoxylin-eosin (H\&E) method. After staining, samples were photographed on an Olympus BX 53 Fluorescence Microscope (Olympus, Japan) for histopathology assay.

\section{TEM analysis}

After collecting and slicing the organ, the specimens were fixed in $2 \%$ glutaraldehyde and osmified with $1 \%$ osmium tetroxide in phosphate buffer at $\mathrm{pH} 7.4$, dehydrated in graded ethanol, embedded in Embed-812 and polymerized for 3 
days. Using a LKB ultramicrotome, 0.5-1.0 $\mu \mathrm{m}$ thick semithin sections were stained with $1 \%$ toluidine blue. Ultrathin sections $(60-90 \mathrm{~nm})$ were then cut with the ultramicrotome and stained for conventional TEM with an automated system (Leica EM Stain; Leica Biosystems, Germany) using uranyl acetate $(5 \mathrm{~g} / \mathrm{L}, 30 \mathrm{~min})$ and lead citrate $(5 \mathrm{~g} / \mathrm{L}, 50 \mathrm{~min})$ at $25^{\circ} \mathrm{C}$. In this study, energy filtered TEM (LIBRA 120; Carl Zeiss, Germany) was used to observe the NP within cells.

\section{Statistical analysis}

All experimental data were presented as the means \pm SE. Data were analyzed by one-way analysis of variance (ANOVA) followed by a two-tailed Student's $t$-test. A $p<0.05$ was considered statistically significant. All analyses were conducted using SPSS for Windows, (ver. 10.0; SPSS, USA).

\section{Results}

\section{Comparison with distribution between HA-NP and HA-polymer}

Although the fluorescence intensity of free Cy5.5 without HA labelling was highest $(65.2 \pm 0.01)$ in liver at $0.5 \mathrm{~h}$, after which was remarkably decreased until day 28 , those levels in all organs examined were almost undetectable after $24 \mathrm{~h}$ (Fig. 1).

As shown in Figure 2, the tissue distribution in entire organs between HA-NP and HA-polymer differs according to the size of the particles. Cy5.5-labeled HA-NPs were mainly detected in liver, kidney and spleen, although some were found in the lung, brain, and testis, and a few were found in the epididymis, submandibular gland, thymus, and heart (Fig. 2A). Although Cy5.5-labeld HA-polymer was found to be highest in the kidney, the fluorescence intensity was remarkably lower than HA-NP (Fig. 2B). With the exception of the liver, kidney, and spleen, HA-polymer levels in other organs such as the lung, brain, testis, epididymis, submandibular gland, thymus, and heart were higher than HA-NP levels, but no significant differences were observed among organs. HApolymer levels in the liver and spleen were expressed at lower intensity than in all other organs. Notably, HA-NP increased slightly in the brain and HA-polymer was highest in the thymus, indicating the need for further study to explain these findings. Overall, HA-NP, which was the smaller particle, had greater distribution in the liver and spleen, while the larger HA-polymer was not.

Temporal distribution pattern of HA-NPs and HApolymers in the main target organs

Figure 3 shows changes in the temporal distribution of each organ during different time periods. HA was initially highly accumulated, but was completely excreted from the liver, spleen, and kidneys 2 weeks post-injection when it was nano-sized. In liver and spleen, the biodistribution pattern was very similar for both HA-NP and HA-polymer, respectively. In the spleen, HA-NP decreased rapidly from $0.5 \mathrm{~h}$ $(1382 \pm 20)$ to day $3(205 \pm 25)$, but increased at day 7 (646

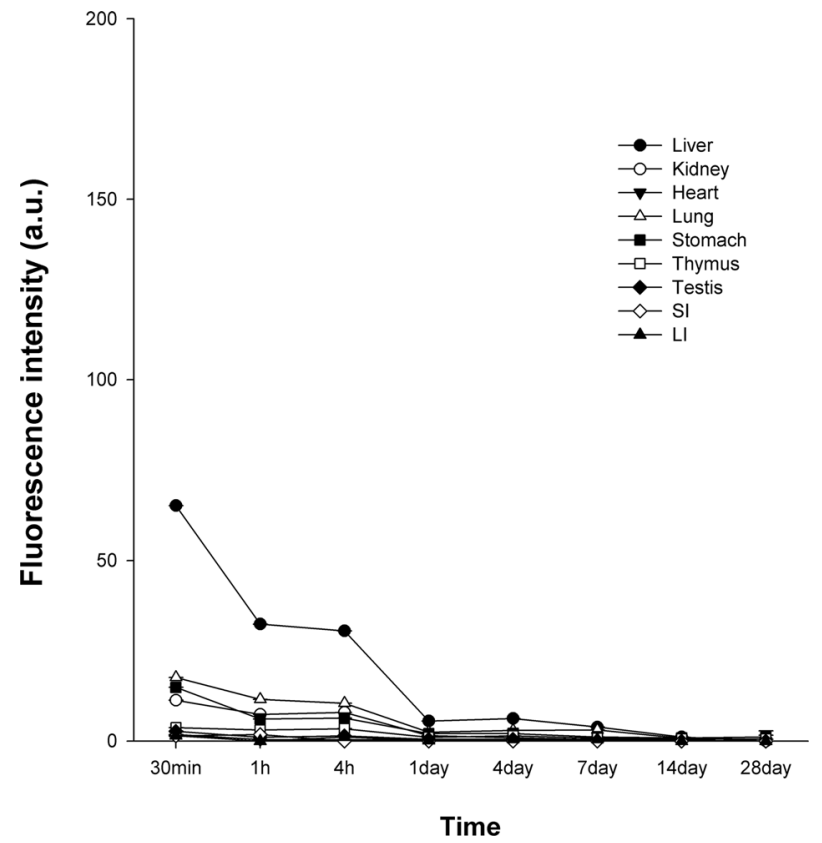

Fig. 1. Fluorescence intensity (106 photon/sec) levels of free Cy5.5 without hyaluronic acid (HA) labelling in kidney, liver, heart, stomach, testis, small intestine (SI), large intestine (LI), thymus, and lung from $0.5 \mathrm{~h}$ to 28 days after the injection. Data are expressed as mean $\pm \operatorname{SE}(n=6)$. a.u., arbitrary unit.

$\pm 24)$ and decreased again at day $28(103 \pm 4)$. HA-polymer in the spleen showed little change from $0.5 \mathrm{~h}(32 \pm 2)$ to day $28(40 \pm 2)$ (Fig. 3A). Similarly, HA-NP in the liver generally decreased from $0.5 \mathrm{~h}(644 \pm 20)$ to day $3(179 \pm 21)$, except for $4 \mathrm{~h}(744 \pm 38)$, then unexpectedly increased at day $7(449 \pm 16)$ and decreased again until day 28 (97 \pm 16$)$. HApolymer in the liver showed no typical changes from $0.5 \mathrm{~h}$ $(109 \pm 5)$ to day $28(140 \pm 2)$ (Fig. 3B). However, the kidneys showed a different pattern of biodistribution. Although HANP was generally detected at higher levels than HA-polymer, both HA-NP and HA-polymer decreased from $0.5 \mathrm{~h}$ (NP: $352 \pm 15$, polymer: $219 \pm 15$ ) to $2 \mathrm{~h}$ (NP: $268 \pm 13$, polymer: $200 \pm 8$ ), then slightly increased at $4 \mathrm{~h}$ (NP: $299 \pm 13$, polymer: $224 \pm 8$ ) and then decreased again until day 28 (NP: $53 \pm 6$, polymer: $48 \pm 3$ ) (Fig. 3C).

\section{Histological examination}

Tissue samples taken at $0.5 \mathrm{~h}, 4 \mathrm{~h}$, day 3 , day 7 , and day 28 from ICR mice injected with Cy5.5-labeled HA-NP and HApolymer were processed for the routine histological examination method and stained with H\&E solution. No tissues (liver, spleen, and kidney) showed pathological changes relative to the control upon microscopic examination (Fig. 4).

Subcellular distribution of HA-NP and HA-polymer TEM images showed particles of Cy5.5-labeled with HA$\mathrm{NP}$ and HA-polymer in the subcellular organelles of each tissue after injection (Fig. 5). HA-NP was observed in the liver, 
A

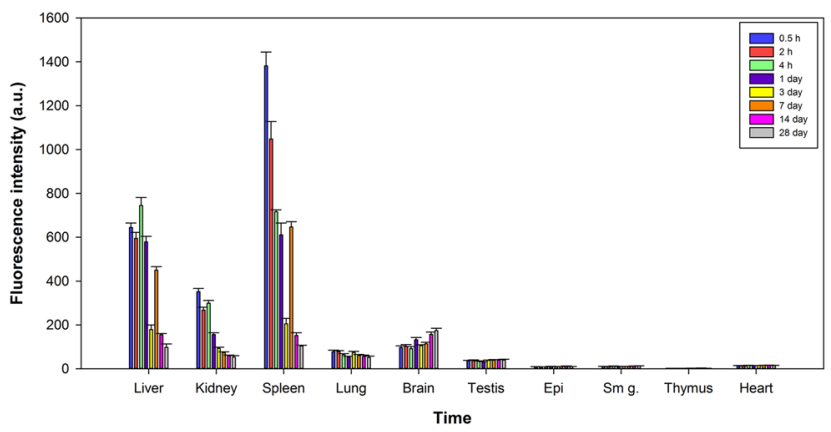

B

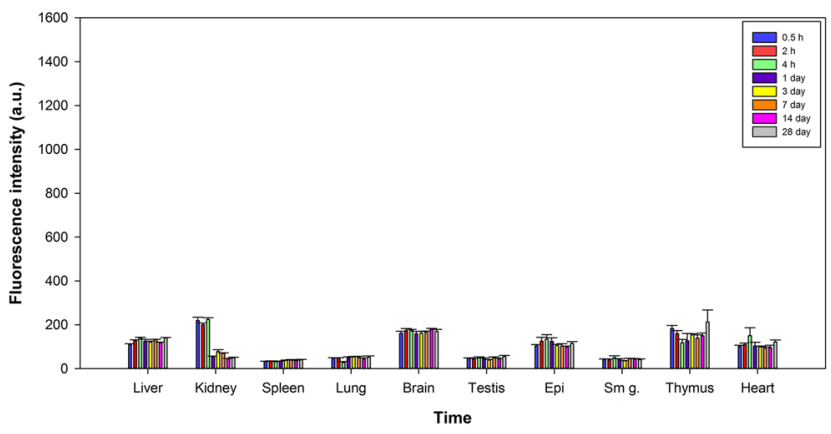

Fig. 2. Biodistribution per unit organ weight in entire organs of cy5.5-labeled HA. HA-nanoparticle (NP) intensity was high in the spleens, livers and kidneys, while it was detected at low levels in other organs (A). When compared with HA-NP, HA-polymer was observed at a very low level in all examined organs $(B)$. Data are expressed as the means \pm SE $(n=6)$. Epi, epididymis; Sm g., submandibular gland.

A

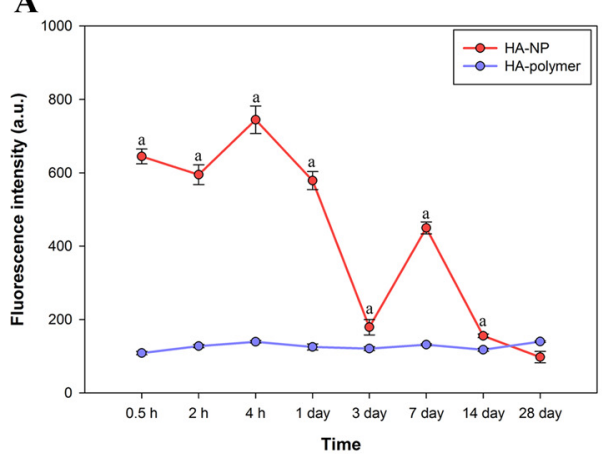

C

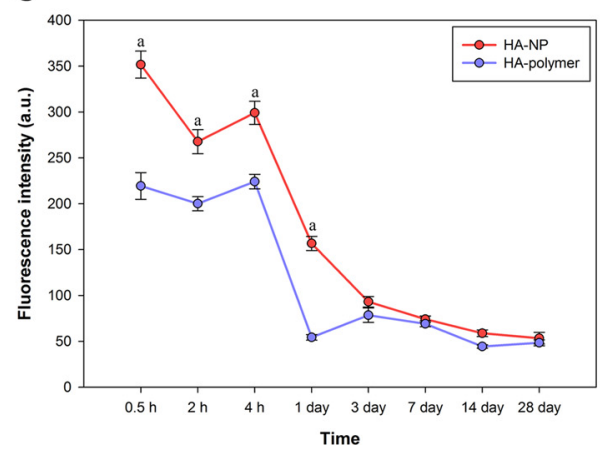

B

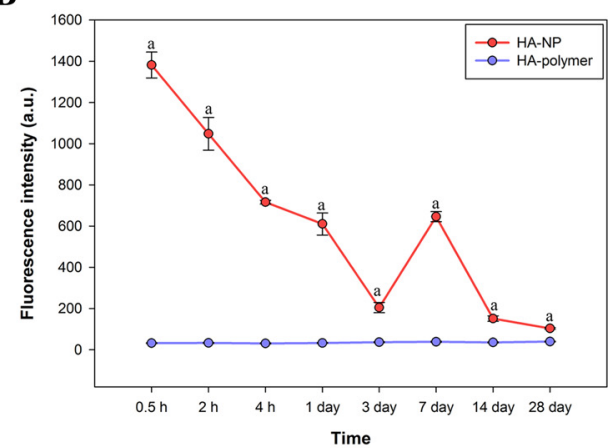

Fig. 3. Temporal tissue distribution per unit organ weight of cy5.5-labeled HA-NP and HA-polymer. A: liver, B: spleen, C: kidney, a: Significantly different $(\mathrm{p}<0.05)$ mean values at the same time. The distribution patterns of HA-NP were similar in the spleen and liver. HA-NP levels in the spleen and liver were remarkably reduced until day 3, but increased sharply between days 3 and 7, then decreased again, while their HA-polymers were not changed at very low levels. In kidneys, both HA-NP and HA-polymer were present at high levels after 30 min of administration and decreased steadily over time. Data are expressed as the means $\pm S E(n=6)$. 

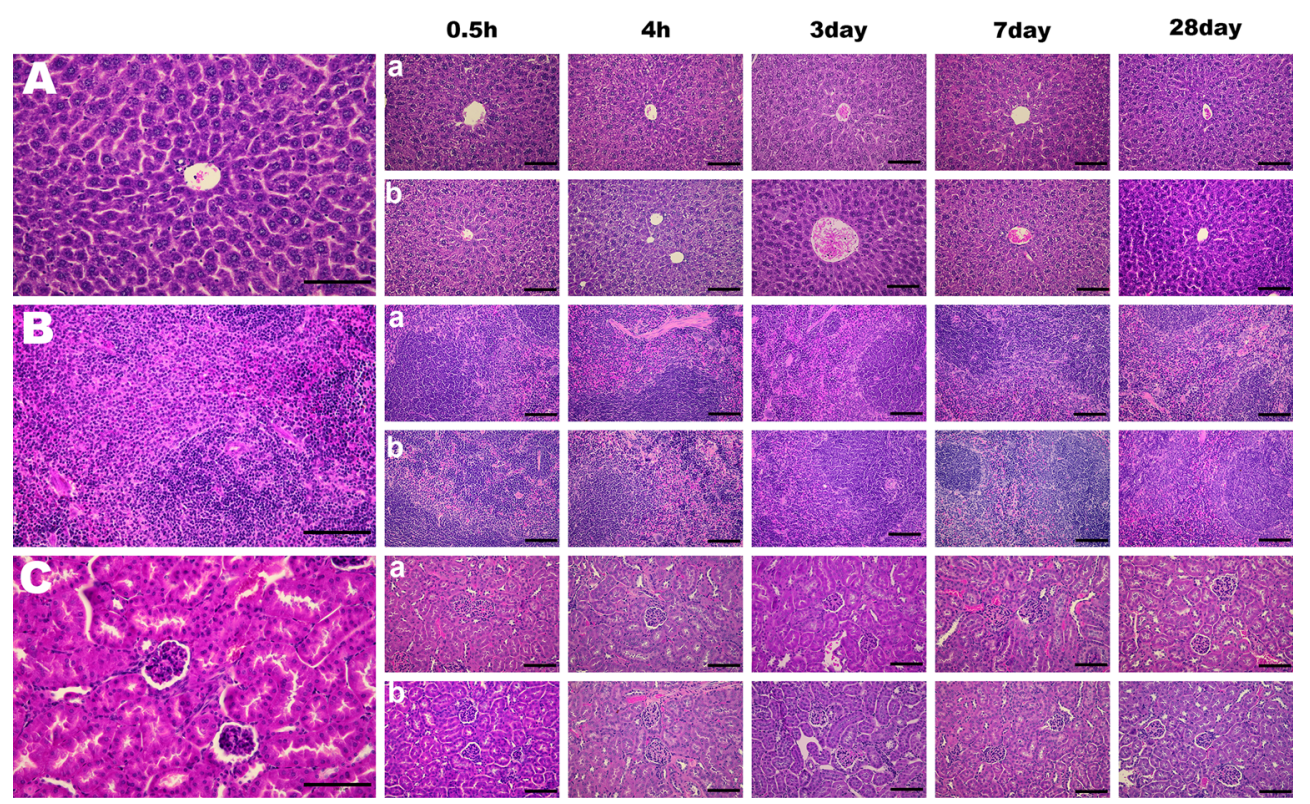

Fig. 4. Representative histological examination of tissues from ICR mice $(n=3)$ injected with HA-NP (a) and HA-polymer (b) through tail intravenous injection over time ( 0.5 h, 4 h, 3 day, 7 day, and 28 day). (A) Liver. (B) Spleen. (C) Kidney. All tissues showed no pathological change, which indicated that neither HA-NP nor HA-polymer caused significant toxicity. H\&E stain. Scale bar $=100 \mu \mathrm{M}$.
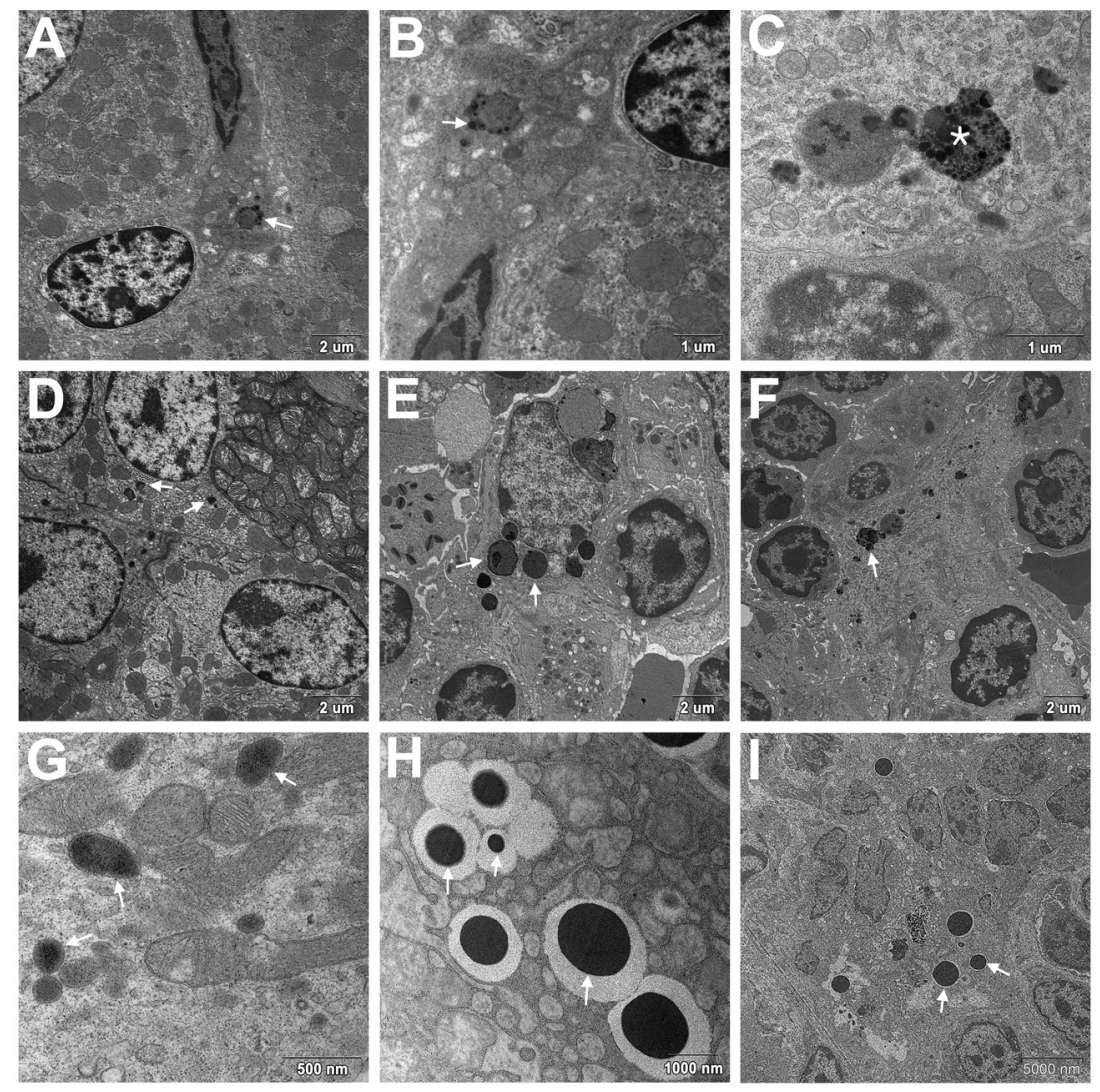

Fig. 5. Representative observations of subcellular structure in tissues from ICR mice $(n=3)$ by transmission electron microscopy. HANP particles (arrows) phagocytosed by Kupffer cells of the liver at day 1 (A and B) after HA-NP injection ( $5 \mathrm{mg} / \mathrm{kg}$ ). Lysosome (asterisk) in kidney macrophages at day 1 (C) and infiltration (arrows) of HA-NP in kidney tubular cells at day 7 (D). Lysosomes (arrows) in the spleen macrophage at day 1 (E and F). Mitochondria (arrows) in spleen white blood cell at day 1 (G). Various HA-polymer particles (arrows) phagocytosed by a macrophage of the spleen at day $28(\mathrm{H}$ and $\mathrm{I})$ after HA-polymer injection $(5 \mathrm{mg} / \mathrm{kg})$. 
spleen, and kidney from day 1 to day 28. In the liver, HA-NP was phagocytosed by Kupffer cells at day 1 (Fig. 5A and B). In the kidney, HA-NP was phagocytosed by macrophages and observed in lysosomes at day 1 (Fig. 5C), after which it accumulated in the cytoplasm of tubular cells at day 7 (Fig. 5D). In the spleen, HA-NP was in the lysosome of macrophages at day 1 (Fig. 5E and $\mathrm{F}$ ) and in mitochondria of white blood cells at day 1 (Fig. 5G). On the other hand, HA-polymers of various sizes, but much larger than HA-NP, were only observed in the spleen at day 28 , where they had been phagocytosed by a macrophage (Fig. $5 \mathrm{H}$ and I).

\section{Discussion}

This study demonstrated the tissue distribution and accumulation of intravenously administered HA-NP in size ranges from $350 \mathrm{~nm}$ to $400 \mathrm{~nm}$ and HA-polymer of larger size in mice. The fluorescence intensity revealed that HANPs were mainly distributed in the liver, spleen, and kidneys, but were also present in the lung, brain and testes, while HA-polymer was distributed to all measured organs in very low levels. These findings are in agreement with previous reports describing the distribution of NPs to multiple organs including the liver, kidneys, heart, lungs, brain, testes, lymph nodes and skin [11]. In other words, distribution of HA-NP appeared to be different from HA-polymer, which consisted of larger particles. HA-NP was mainly distributed in the spleen, followed by the liver and kidneys, whereas HA-polymer was mainly found in the kidney and hardly appeared in the liver and spleen. In the different organs examined, no significant difference by sizes was detected. This tissue distribution difference between HA-NP and HApolymer was investigated over the entire period of the experiment. HA biodistribution was considered to be related to particle size. It has been suggested that tissue distribution by HA sizes is proportional to toxicity and health [10]. In the future, we need to know when and why the kinetics and toxicity of nanoparticles are expressed.

In the present study, most nanoparticles were predominantly found in the liver and spleen, which are representative organs with the reticuloendothelial system that plays a major role in removing certain toxic substances from the blood. Accumulation of nanomaterials like silica, gold and iron oxide, titanium dioxide, and quantum dots was mainly detected in the liver and spleen. Moreover, the size-related distribution over these organs suggested that organs with permeable endothelial linings mainly in blood vessels such as the liver and spleen play a role significantly to the uptake of nanomaterials. In addition, particles smaller than $100 \mathrm{~nm}$ accumulate in the liver, but larger particles accumulate mainly in the spleen [5, 18, 26-28], which is in agreement with our findings that HA-NP of $320-400 \mathrm{~nm}$ were mainly distributed in the spleen. For all time points examined in the liver, spleen and kidney, HA-NP decreased remarkably compared to HA-polymer. Possible explanations for this finding may be faster degradation, elimination, and excretion of HANP than HA-polymer.

The different biodistribution of HA-NP and HA-polymer was also probably correlated with different capture features in various tissues and the in vivo degradation tendency of HA. The initial decreasing distribution in the first 3 days can primarily be due to the rapid degradation of HA-NP [24]. However, within 7 days of administration, the re-distribution of the fluorescence intensity in the liver and spleen could have been attributed to the continuous capture and reduced degradation rates of HA-NP, which probably resulted in their consecutive uptake and increased organic accumulation. These findings indicate that the HA-NP degradation can be remarkably inhibited by increasing the HA-NP accumulation [3]. This also implies that there are always uncaptured HA-NP remaining and circulating in the blood. Moreover, when compared with HA-NP, HA-polymer was not captured by the liver and spleen, implying that HA-polymer has a longer blood-circulation lifetime [5]. Within 28 days of injection, the biodistribution of HA-NP in the liver and spleen decreased again relative to day 7 , which was likely due to their slow, but continued degradation. Although HA-NP re-increased suddenly at 7 days after administration, but no definite reason was known so far, indicating the need for further study to explain these findings. In addition, most HA-NP could be degraded completely for prolonged time periods. Interestingly, distributions of HA-polymer in the liver and spleen from 30 min to 28 days after injection were especially lower than in all other organs, which might have been because of the difficulty of their biodegradation and/or transfer to other tissues. For kidneys, the distribution kept decreasing with time from $30 \mathrm{~min}$ to 28 days, probably because the majority of these nanoparticles had been captured and excreted from blood to urine in the first $30 \mathrm{~min}$ (or longer) after injection, resulting in the excretion rate of degraded HA being higher than their accumulation rates beyond 30 min post-injection [8].

Histological analyses were conducted to investigate tissue toxicity with organ accumulation after injection of HA-NP and HA-polymer. The liver, spleen, and kidney, where the HA-NP and HA-polymer were mainly distributed did not show any pathological changes, indicating that neither $5 \mathrm{mg} /$ $\mathrm{kg}$ of HA-NP nor HA-polymer cause significant tissue toxicity, and that both HA-NP and HA-polymer possess good tissue compatibility [8]. Such compatibility can be attributed to the stable physicochemical properties, biodegradability, and biocompatibility of the HA particles.

To directly identify the biodistribution of exogenous HA, the main organs in which HA-NP and HA-polymer were distributed (liver, spleen, and kidneys) were subjected by ultrastructural analyses using TEM. HA-NP was observed in Kupffer cells of the liver and in white blood cells of the spleen, indicating that it was moved and engulfed through the blood by phagocytosis of the liver and spleen [3]. On the other hand, HA-polymers of various sizes, but much larger than HA-NP, were only observed in the spleen at day 28 , 
where they had been phagocytosed by a macrophage. Interestingly, recent studies showed that these findings may be due to cellular uptake of the HA-NPs by phagocytic cells of the reticuloendothelial system and by liver sinusoidal endothelial cells expressing the hyaluronic acid receptor for endocytosis [22, 29]. HA-NP also appeared to be excreted through the kidneys [8], as indicated by its being found in lysosomes of the phagocyte and tubules in kidneys.

In summary, HA was initially highly accumulated, but was completely excreted from the liver, spleen, and kidneys at 2 weeks post-injection when it was nano-sized. These findings suggest that HA-NP could be considered a desirable drug carrier if the target organs of the drug are the liver, kidney, and spleen.

\section{Acknowledgments}

Authors wish to thank Dr. Kwangmeyung Kim of the Korea Institute of Science and Technology who kindly provided HA-polymers and HA-NPs. This research was supported by the Basic Science Research Program (2016R1D1A1B01015625) through the National Research Foundation of Korea (NRF) funded by the Ministry of Education, Science and Technology, Republic of Korea.

\section{References}

1. Alexis F, Pridgen E, Molnar LK, Farokhzad OC. Factors affecting the clearance and biodistribution of polymeric nanoparticles. Mol Pharm 2008, 5, 505-515.

2. Balazs EA, Denlinger JL. Clinical uses of hyaluronan. Ciba Found Symp1989, 143, 265-275.

3. Bazile D, Prud'homme C, Bassoullet MT, Marlard M, Spenlehauer G, Veillard M. Stealth Me.PEG-PLA nanoparticles avoid uptake by the mononuclear phagocytes system. J Pharm Sci 1995, 84, 493-498.

4. Choi KY, Chung H, Min KH, Yoon HY, Kim K, Park JH, Kwon IC, Jeong SY. Self-assembled hyaluronic acid nanoparticles for active tumor targeting. Biomaterials 2010, 31, 106-114.

5. De Jong WH, Hagens WI, Krystek P, Burger MC, Sips AJAM, Geertsma RE. Particle size-dependent organ distribution of gold nanoparticles after intravenous administration. Biomaterials 2008, 29, 1912-1919.

6. Ferrari M. Cancer nanotechnology: opportunities and challenges. Nat Rev Cancer 2005, 5, 161-171.

7. Hanahan D, Weinberg RA. The hallmarks of cancer. Cell 2000, 100, 57-70.

8. He Q, Zhang Z, Gao F, Li Y, Shi J. In vivo biodistribution and urinary excretion of mesoporous silica nanoparticles: effects of particle size and PEGylation. Small 2011, 7, 271280.

9. Jain KK. Current status and future prospects of drug delivery systems. Methods Mol Biol 2014, 1141, 1-56.

10. Kakehi K, Kinoshita M, Yasueda S. Hyaluronic acid: separation and biological implications. J Chromatogr B Analyt Technol Biomed Life Sci 2003, 797, 347-355.

11. Kim YS, Kim JS, Cho HS, Rha DS, Kim JM, Park JD,
Choi BS, Lim R, Chang HK, Chung YH, Kwon IH, Jeong J, Han BS, Yu IJ. Twenty-eight-day oral toxicity, genotoxicity, and gender-related tissue distribution of silver nanoparticles in Sprague-Dawley rats. Inhal Toxicol 2008, 20, 575-583.

12. Kinzler KW, Vogelstein B. Landscaping the cancer terrain. Science 1998, 280, 1036-1037.

13. Kumari A, Yadav SK, Yadav SC. Biodegradable polymeric nanoparticles based drug delivery systems. Colloids Surf B Biointerfaces 2010, 75, 1-18.

14. Lankveld DPK, Oomen AG, Krystek P, Neigh A, Troostde Jong A, Noorlander CW, Van Eijkeren JCH, Geertsma RE, De Jong WH. The kinetics of the tissue distribution of silver nanoparticles of different sizes. Biomaterials 2010, 31, 8350-8361.

15. Lapčik L Jr, Lapčik L, De Smedt S, Demeester J, Chabreček P. Hyaluronan: preparation, structure, properties, and applications. Chem Rev 1998, 98, 2663-2684.

16. Lee H, Lee K, Park TG. Hyaluronic acid-paclitaxel conjugate micelles: synthesis, characterization, and antitumor activity. Bioconjug Chem 2008, 19, 1319-1325.

17. Lee MJE, Veiseh O, Bhattarai N, Sun C, Hansen SJ, Ditzler S, Knoblaugh S, Lee D, Ellenbogen R, Zhang M, Olson JM. Rapid pharmacokinetic and biodistribution studies using cholorotoxin-conjugated iron oxide nanoparticles: a novel non-radioactive method. PLoS One 2010, 5, e9536.

18. Ma HL, Xu YF, Qi XR, Maitani Y, Nagai T. Superparamagnetic iron oxide nanoparticles stabilized by alginate: pharmacokinetics, tissue distribution, and applications in detecting liver cancers. Int J Pharm 2008, 354, 217-226.

19. Mironov V, Kasyanov V, Zheng Shu X, Eisenberg C, Eisenberg L, Gonda S, Trusk T, Markwald RR, Prestwich GD. Fabrication of tubular tissue constructs by centrifugal casting of cells suspended in an in situ crosslinkable hyaluronan-gelatin hydrogel. Biomaterials 2005, 26, 76287635.

20. Peer D, Karp JM, Hong S, Farokhzad OC, Margalit R, Langer R. Nanocarriers as an emerging platform for cancer therapy. Nat Nanotechnol 2007, 2, 751-760.

21. Peer D, Margalit R. Loading mitomycin C inside long circulating hyaluronan targeted nano-liposomes increases its antitumor activity in three mice tumor models. Int $\mathrm{J}$ Cancer 2004, 108, 780-789.

22. Platt VM, Szoka FC Jr. Anticancer therapeutics: targeting macromolecules and nanocarriers to hyaluronan or CD44, a hyaluronan receptor. Mol Pharm 2008, 5, 474-486.

23. Soppimath KS, Aminabhavi TM, Kulkarni AR, Rudzinski WE. Biodegradable polymeric nanoparticles as drug delivery devices. J Control Release 2001, 70, 1-20.

24. Tamaki S, Ueno T, Torimura T, Sata M, Tanikawa K. Evaluation of hyaluronic acid binding ability of hepatic sinusoidal endothelial cells in rats with liver cirrhosis. Gastroenterology 1996, 111, 1049-1057.

25. Toole BP. Hyaluronan: from extracellular glue to pericellular cue. Nat Rev Cancer 2004, 4, 528-539.

26. Wang J, Zhou G, Chen C, Yu H, Wang T, Ma Y, Jia G, Gao Y, Li B, Sun J, Li Y, Jiao F, Zhao Y, Chai Z. Acute toxicity and biodistribution of different sized titanium dioxide particles in mice after oral administration. Toxicol Lett 2007, 168, 176-185. 
27. Xie G, Sun J, Zhong G, Shi L, Zhang D. Biodistribution and toxicity of intravenously administered silica nanoparticles in mice. Arch Toxicol 2010, 84, 183-190.

28. Yang RS, Chang LW, Wu JP, Tsai MH, Wang HJ, Kuo YC, Yeh TK, Yang CS, Lin P. Persistent tissue kinetics and redistribution of nanoparticles, quantum dot 705 , in mice:
ICP-MS quantitative assessment. Environ Health Perspect 2007, 115, 1339-1343

29. Zhou B, Weigel JA, Fauss L, Weigel PH. Identification of the hyaluronan receptor for endocytosis (HARE). J Biol Chem 2000, 275, 37733-37741. 